\title{
Dosimetry and Treatment Descriptions Using the First Completely Automated Stereotactic Intracranial Radiosurgery Rotating Gamma Ray Unit in America
}

\author{
Eduardo E. Lovo ${ }^{1}$, Fidel J. Campos ${ }^{1}$, Victor E. Caceros ${ }^{1}$, Mario H. Minervini ${ }^{1}$, William A. Reyes ${ }^{1}$ \\ 1. Radiosurgery Program, Centro Internacional De Cancer, Hospital De Diagnostico, San Salvador, SLV
}

Corresponding author: Eduardo E. Lovo, lovoiglesias@gmail.com

\section{Abstract \\ Introduction}

The objective is to report the dosimetry and safety profiles of the first fully automatized rotating gamma ray unit for intracranial radiosurgery in America.

\section{Methods}

Dosimetry tests were conducted by our institution using the standard of examination and calibration and the Intelligent $\gamma$ Radiometer of the China Research Institute of Measurement. The phantom and dosimetry tests were performed by the Outreach Physics Section of MD Anderson Cancer Center and the Anchorage Radiation Therapy Center using the Radiation Therapy Oncology Group (RTOG) radiosurgery quality assurance guidelines. Clinically, 233 patients were treated.

\section{Results}

Mechanical precision was $0.16 \mathrm{~mm}$ and the offset registered at the phantom on all axes was 0.0 . The ratio of the dose to the center was 0.97 (0.95-1.05), the ratio of the treated volume was $0.95(0.75-1.25)$, the ratio of the measured treated volume to the volume of the target was $1.29(1.00-2.00)$, the ratio of the minimal dose to the dose prescribed was $1.05(>0.90)$, with a treated volume of $0.95(0.75-1.25)$ and a minimum dose to target of $1.05(>0.90)$. The dose rate at loading was $3.89 \mathrm{~Gy}$ per minute. None of the patients treated experienced severe complications.

\section{Conclusions}

The dosimetry studies are compliant with quality assurance standards for intracranial radiosurgery.

Received 02/28/2018

Review began 03/20/2018 Review ended 04/23/2018 Published 04/27/2018

๑) Copyright 2018 Lovo et al. This is an open access article distributed under the terms of the Creative Commons Attribution License CC-BY 3.0., which permits unrestricted use, distribution, and reproduction in any medium, provided the original author and source are credited.
Categories: Medical Physics, Radiation Oncology, Neurosurgery

Keywords: radiosurgery, stereotactic radiosurgery, gamma-knife, brain, dosimetric

\section{Introduction}

In accordance with the American Society for Therapeutic Radiology and Oncology (ASTRO) and others, part of the definition of stereotactic radiosurgery is, "it is a discipline that utilizes externally generated ionizing radiation to inactivate or eradicate (a) defined target(s) in the head or spine without the need to make an incision, while defining the target by high-resolution imaging" [1].

Intracranial stereotactic radiosurgery was developed by Lars Leksell and the tool he and his group developed: the Gamma knife. Since 1968, this has been demonstrated to be extremely precise $(<1 \mathrm{~mm})$ and capable of producing a steep dose gradient of radiation that is, till date, the gold standard and to what, typically, other radiosurgery systems compare to [2]. In the evolution of this technology, the amount of cobalt 60 sources reduced slightly from 201 in model U to 192 in Perfexion (Elekta Instruments AB, Stockholm, Sweden). The main advances have been automation as well as intracranial reach, which increased dramatically according to some authors [3]. Image guidance is also available now, which allows multisession radiosurgery.

During the 1990s, other companies in Asia begun developing intracranial stereotactic gamma ray machines that rotated the cobalt 60 sources in relation to the isocenter, needing a significantly lower number of sources (25 or 30) with similar dosimetry characteristics than the static Gamma knife units [4-5] of the time. In 2014, the first fully automated rotating gamma ray unit called Infini (Masep Medical Company, Shenzhen, China) came into service in the American continent and 233 patients have been treated so far.

The purpose of the current communication is to report the basic dosimetry and patient treatment process to 


\section{Materials And Methods}

The current investigation was carried out from March 2014 to January 2017. During this period, 30 cobalt 60 sources were loaded on a fully automated, rotating gamma-ray system called Infini. Dosimetry studies were carried out by the institution where Infini was installed and three outside institutions participated in a phantom test analysis before the first patient was treated. During this time, 233 patients were treated for different pathological entities affecting the brain.

\section{Machine characteristics}

Infini is a fully automated, rotating gamma-ray machine designed for intracranial stereotactic radiosurgery (Figure 1). It uses 30 rotating cobalt 60 sources that revolve with regards to the mechanical isocenter using a multisource, non-isometric spiral focusing method. These cobalt sources are arranged in six rows of five cobalt sources each and rotate at a one rotation per minute speed. It has four different sizes of collimators $(4,8,14,18 \mathrm{~mm})$ to choose from, as well as three different angles that can be used, 70,90 , and $110^{\circ}$, in accordance with the head holder and the trunnion of the machine. The intracranial reach is $180 / 180 / 230 \mathrm{~mm}$ in the X/Y/Z axis, respectively. The operations of the collimators and patient positioning are completely automated, allowing a seamless workflow. Finally, it has an independent beam switch that can turn on or off the passage of radiation every 5 degrees.

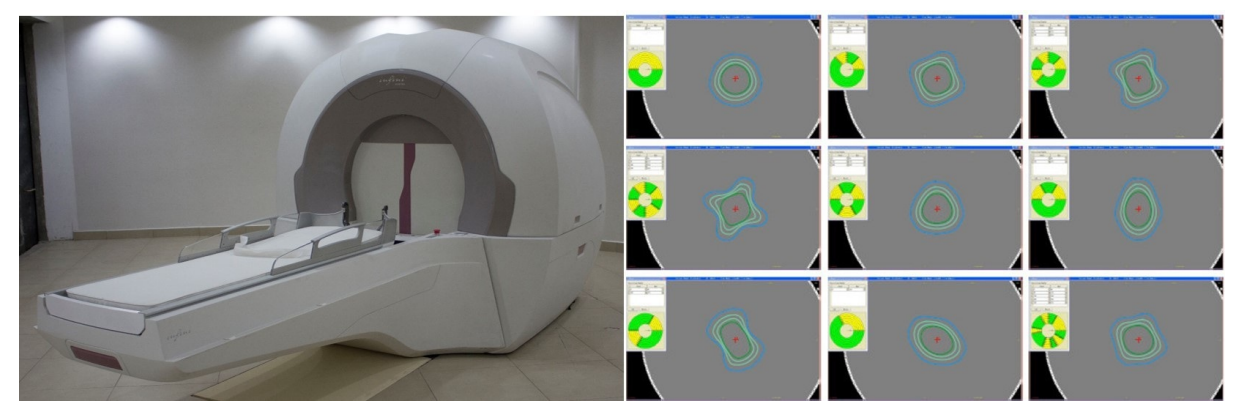

FIGURE 1: View of Infini and some dose sculpturing capabilities

Composite image: To the left is an oblique view of Infini (Masep Medical Company, Shenzhen, China); to the right are different dose configurations that can be achieved by selectively blocking different sectors at 5 degrees or more.

\section{Dosimetry evaluation process}

Dosimetry results were obtained from final commissioning by the local institution and the manufacturing company using the standard of examination and calibration of the Intelligent $y$ Radiometer of the China Research Institute of Measurement. Also, a stereotactic radiosurgery head phantom irradiation test was performed and audited by the Outreach Physics Section of Imaging and Radiation Oncology Core (IROC), an institution that belongs to MD Anderson Cancer Center (Houston, TX, USA), as follows: An anthropomorphic head phantom with a $1.9 \mathrm{~cm}$ diameter spherical target was mounted on the stereotactic frame and was irradiated with a single isocenter with a maximum dose to the target of $30 \mathrm{~Gy}$. The doses to two specific points were measured using thermoluminescent dosimetry (TLD) capsules and the dose distribution was evaluated using two orthogonal sheets of radiochromic film.

The dose rate was confirmed by the Anchorage Radiation Therapy Center by irradiation and taking readings from the center of the $16 \mathrm{~cm}$ polystyrene phantom using an $18 \mathrm{~mm}$ collimator utilizing the TG-21 American Association of Physicist in Medicine (AAPM) protocol to determine the dose rate. The dosimetry equipment used was an ionization chamber, Exradin (A1SL SN XW 060580) and an electrometer, Standard Imaging MAX-4000 (SN 131014).

\section{Patient treatment flow}

At the time of this report, 233 patients had been treated with diverse intracranial lesions and neurological and psychiatric conditions (Table 1). In brief, the process regarding patient treatment is as follows: The patient is admitted by an anesthesiologist that provides slight sedation using intravenous fentanyl, as needed. At the same time, $8 \mathrm{mg}$ of decadron (Dexamethasone) is administered intravenously. The stereotactic frame is placed while the patient is sedated under local anesthesia using a mixture of 50/50 lidocaine and bupivacaine at the sites where the pins will pierce the skin into the external bone cortex of the cranium. Immediately after the frame has been secured to the patient, the $\mathrm{N}$ reference box is fixed to the frame and an MR head holder adaptor and antenna are fixed in the MRI unit, Avanto 1.5 Tesla (Siemens 


\section{Cureus}

Corporation, Erlangen, Germany). The following sequences are carried out: one T2, 6-mm axial sequence at $0^{\circ}$ of the full head, which is later used in the Superplan (Masep Medical Co., Shenzhen, China) treatment planning system (TPS) to provide the volume of the patients' skin, one T1-gadolinium 1-mm axial and one coronal sequence of the area of interest at $0^{\circ}$ and $90^{\circ}$ respectively is carried out in cases where tumors need to be identified. In cases of metastasis, the T1 is done with a double dose of gadolinium for the entire head and acquired at 1-mm axial slices. When AVMs are being treated, we also use an axial T2 CISS of $1 \mathrm{~mm}$ of the region of interest in addition to the T1-gadolinium. The same sequence is used when detail is needed of the intracranial nerves. When functional targets inside the brain are being chosen, a T1 without gadolinium or CISS and fluid attenuation inversion recovery (FLAIR) sequences with 1-mm slice widths are used to visualize internal capsules or other structures, such as anterior and posterior commissures. All sequences required a field of view (FOV) of 240 for proper visualization on the TPS. More recently, we performed image fusion (two stereotactic image sets fused using the reference box marks as guides) with CT and used this as the primary study in cranium base tumors or patients where an MRI is not feasible when patients are overweight or have pacemakers.

\begin{tabular}{|c|c|c|c|c|}
\hline Pathology & $\begin{array}{l}\text { Number of } \\
\text { treatments }\end{array}$ & Age & Gender & Mean Marginal dose \\
\hline $\begin{array}{l}\text { Metastasis and } \\
\text { malignant glioma }\end{array}$ & $41(17.5 \%)$ & $\begin{array}{l}57.6(7- \\
78)\end{array}$ & $\begin{array}{l}119(51 \%) \text { Female } 114(49 \%) \text { Male. For } \\
\text { whole series }\end{array}$ & $\begin{array}{l}21 \text { Gy }\left(10-24 \text { Gy) to the } 56 \% \text { IDL* }^{*}\right. \\
(49-90 \%)\end{array}$ \\
\hline Benıgn tumor & $97(41.6 \%)$ & $\begin{array}{l}52(28- \\
76)\end{array}$ & & $\begin{array}{l}14 \text { Gy }(10-22 \text { Gy) to the } 50 \% \text { IDL } \\
(49-52 \%)\end{array}$ \\
\hline Vascular pathology & $43(18.3 \%)$ & $\begin{array}{l}26(16- \\
67)\end{array}$ & & 18 Gy $(16-20$ Gy) to the $50 \%$ IDL \\
\hline Functional & $52(22.3 \%)$ & $\begin{array}{l}59(31- \\
80)\end{array}$ & & $\begin{array}{l}100 \text { Gy (75-150 Gy) to the } 100 \% \\
\text { IDL }\end{array}$ \\
\hline
\end{tabular}

\section{TABLE 1: Pathology and treatment characteristics}

Treatment characteristic of the whole series shown by general classifications of pathologies, patient characteristics and mean marginal dose.

* IDL- Isodose lines

The images were transferred to TPS via the ethernet. Regions of interest (ROI), as well as the Planned Target Volume (PTV) or target, are generally contoured by the neurosurgeon in collaboration with the radiation oncologist. The final plan is configured by the physicist with the participation of the neurosurgeon and radiation oncologist. Once it has been approved, the patient is carried into the Infini and fixed to the treatment bed with the head frame holder to the trunnion on the treatment couch and the angle of treatment is verified so that the irradiation can begin. After the treatment is finished, the frame is removed from the patient and they are instructed to remove the circular band-aids placed at the pin sites 24 hours after the treatment. They are discharged immediately after treatment.

\section{Results}

The mechanical precision of the machine was $0.16 \mathrm{~mm}$, on average, for all the collimators tested ( 0.11 for the $4 \mathrm{~mm}, 0.16$ for the $8 \mathrm{~mm}, 0.17$ for the $14 \mathrm{~mm}$, and 0.20 for the $18 \mathrm{~mm}$ ). The offset registered at the head phantom irradiation test by MDACC with a prescription dose of $15 \mathrm{~Gy}$ to the $50 \%$ isodose line on all axes was 0.0 , as measured along the different profiles.

The precision of beam alignment is determined by comparing the measured dose profiles of the resulting dose distribution located in a volume surrounding the radiological focus point with the calculated dose profiles, assuming identical geometrical and radio physical conditions. An acceptance criterion with a deviation between dose profiles of no more than $-+1 \mathrm{~mm}$ at the $50 \%$ isodose line, it was accomplished with all the dose profiles in accordance to manufacturer specifications (Figure 2). 


\section{Cureus}
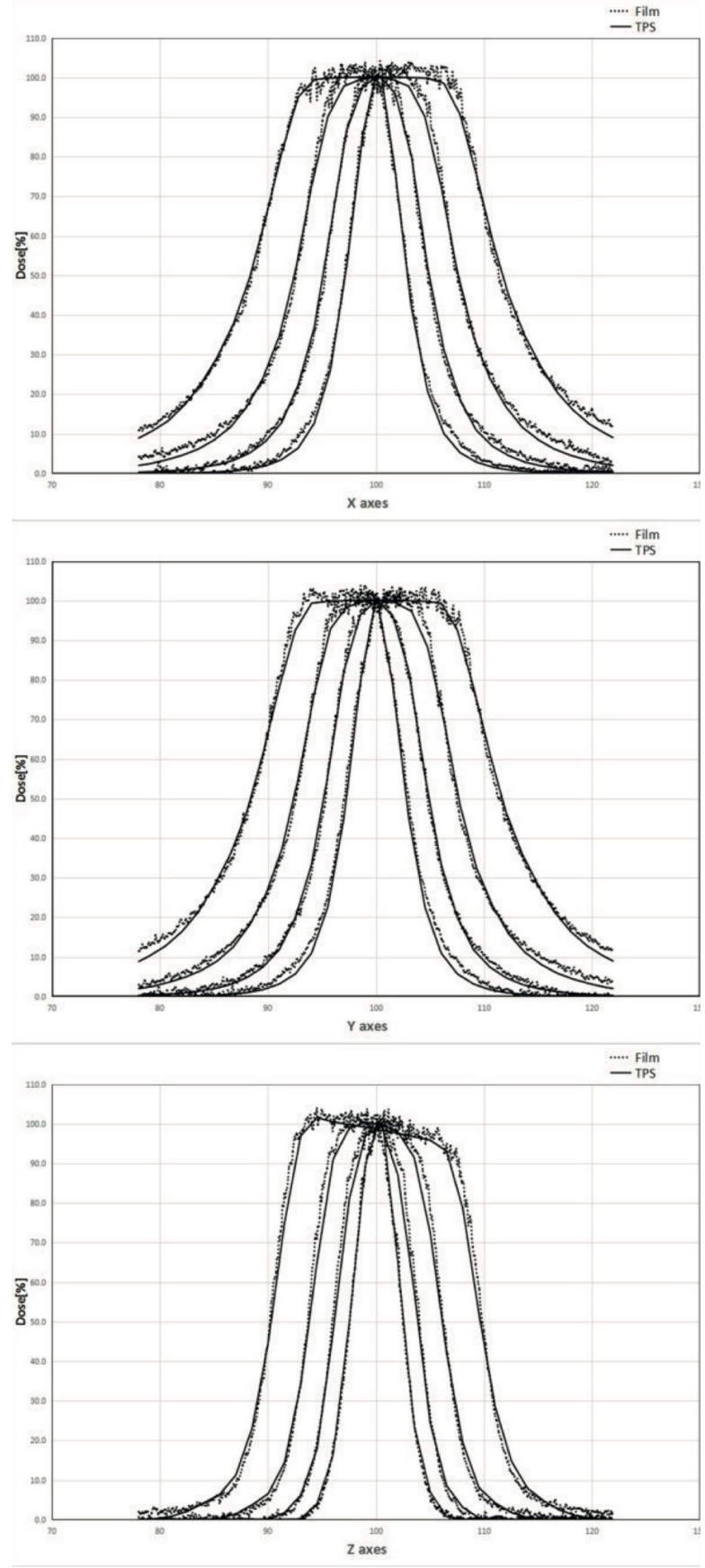

Figure 2

\section{FIGURE 2: Beam profiles for different collimator sizes}

Profiles calculated from different-sized collimators - 4, 8, 14, and $18 \mathrm{~mm}$ - for all stereotactic $X, Y$, and $Z$ axes. On top is the $X$ axis, in the middle is the $Y$ axis, and at the bottom of the image is the $Z$ axis.

The initial dose rate at the isocenter was $3.89 \mathrm{~Gy}$ per minute using the $18 \mathrm{~mm}$ collimator. The results of the head anthropomorphic phantom irradiation test were the following: The ratio of the dose to the center of the target measured by IROC to the dose reported by the local institution was 0.97 (acceptance criteria: 0.951.05 ), the ratio of treated volume taken from the films' evaluation was 0.95 (acceptance criteria: $0.75-1.25$ ), the ratio of the measured treated volume to the volume of the target was 1.29 (acceptance criteria: 1.002.00 ), and, finally, the ratio of the minimal dose to the dose prescribed was 1.05 (acceptance criteria $>0.90$ ), with a treated volume of 0.95 (criteria: 0.75-1.25) with a minimum dose to target of 1.05 (criteria >0.90).

Patient and treatment characteristics can be seen in Table 1 . Thus far, out of the 233 patients treated, most 
were female - 119 (51\%) with 114 male (49\%), the median age was 50.3 years (7-80), 138 patients (59.2\%) were treated for malignant or benign tumors, 43 patients (18.3\%) were treated for vascular pathology, and 52 others (22\%) were treated under functional radiosurgery protocols for trigeminal neuralgia, thalamotomy, hypophysectomy, and anterior capsulotomy (Figure 3).

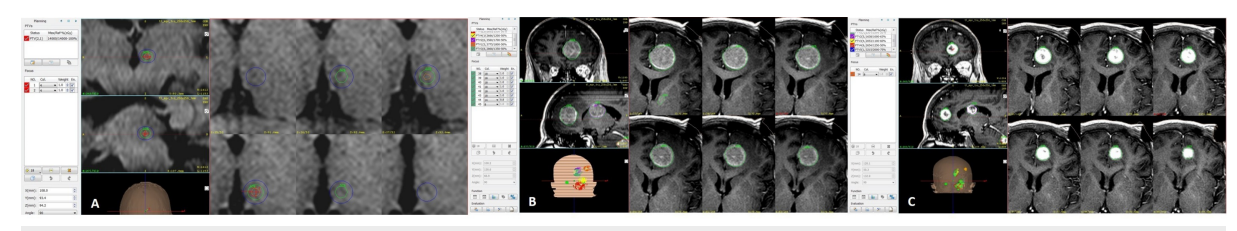

\section{FIGURE 3: Examples of clinical applications}

A. Dosimetry and isodose lines of a medial thalamotomy plan for intractable contralateral pain from trigeminal neuralgia. The inner isodose line represents the $75 \%$ curve of the total dose, the middle isodose line represents the $50 \%$, and the outer isodose line represents $25 \%$ of 140 Gy of the prescription dose. B. and C. are two different treatments plans for a large cerebral metastasis spaced by $\mathbf{3 0}$ days in a protocol for dose escalation. In each treatment, a prescription dose of $1350 \mathrm{cGy}$ to the $50 \%$ and $53 \%$ isodose lines was used, respectively, with a median dose of $1942 \mathrm{cGy}$ per treatment.

The mean follow-up was 26.2 months (4-39) and only two cases (0.8\%) experienced Radiation Therapy Oncology Group (RTOG) toxicity exceeding grade 1; both were in brain metastases cases caused by radiation necrosis.

\section{Discussion}

Although the mechanical precision of older models of rotating gamma ray units had already been evaluated in comparison to the gamma knife models of that time and deemed similar [4-5], this is the first report of the radiological accuracy of a fully automatized rotating gamma ray unit known as Infini. Since this model is a relatively novel intracranial stereotactic radiosurgery unit and few exist in the world at this moment, it is necessary to report from the dosimetry point of view that it is compliant with regards to precision and beam irradiation characteristics with the RTOG guidelines [6].

As demonstrated by dosimetry results, Infini is comparable to the Perfexion and older versions of gamma knife. One difference is the higher initial cobalt 60 activity $\left(2.4 \times 10_{14} \mathrm{~Bq}\right)$ at the loading of the Infini that gave it a greater initial dose rate ( 3.89 Gy per minute) than the one reported by Perfexion [7]; thus, possibly traducing in faster treatment times despite the small amount of cobalt 60 sources. One of the clearest advantages of the rotating principle is the need for a small number of cobalt sources (30 vs. 192) that transduces into lower costs and higher efficiency. This aspect was very important for underdeveloped countries such as the one where the system was installed.

At the time of this report, over 233 patients have been treated. Although it is not the intention of this report to evaluate the clinical results of the diverse pathologies treated, enough follow-up has been done to say that the treatment is safe and has low complications associated with the procedure, even under extreme uses, such as functional diseases where very high doses of radiation are delivered to small areas in very eloquent regions of the brain.

\section{Conclusions}

Infini is a completely automatized intracranial stereotactic machine that uses 30 cobalt sources in an array that circulates around the isocenter and thus the target. The dosimetry studies and clinical use has demonstrated that it is safe and compliant with the RTOG quality assurance standards for intracranial radiosurgery.

\section{Additional Information \\ Disclosures}

Human subjects: Consent was obtained by all participants in this study. Comite de Etica e Investigacion Centro Internacional de Cancer issued approval NA. The Ethics and Research Committee of the Centro Internacional de Cancer certifies that the study entitled Dosimetry and Treatment Descriptions Using the First Completely Automated Stereotactic Intracranial Radiosurgery Rotating Gamma Ray Unit in America had full written consent for treatment by all the patients reported and that the treatments that were performed were in accordance to international recommendations for intracranial radiosurgery. . Animal subjects: All authors have confirmed that this study did not involve animal subjects or tissue. Conflicts of interest: In compliance with the ICMJE uniform disclosure form, all authors declare the following: Payment/services info: All authors have declared that no financial support was received from any organization for the submitted work. Financial relationships: All authors have declared that they have no 


\section{Cureus}

financial relationships at present or within the previous three years with any organizations that might have an interest in the submitted work. Other relationships: All authors have declared that there are no other relationships or activities that could appear to have influenced the submitted work.

\section{References}

1. Seung SK, Larson DA, Galvin JM, et al.: American College of Radiology (ACR) and American Society for Radiation Oncology (ASTRO) practice guideline for the performance of stereotactic radiosurgery (SRS). Am J Clin Oncol. 2013, 36:310-315. 10.1097/COC.0b013e31826e053d

2. Chin LS, Robert B, King MG: Principles and Practice of Stereotactic Radiosurgery. William F. Regine (ed): Springer Verlag, New York; 2008. 2-3. 10.1007/978-1-4614-8363-2

3. Lindquist C, Paddick I: The Leksell Gamma Knife Perfexion and comparisons with its predecessors . Neurosurgery. 2008, 2:721-732. 10.1227/01.neu.0000289726.35330.8a

4. Goetsch SJ, Murphy BD, Schmidt R, Micka J, De Werd L, Chen Y, Shockley S: Physics of rotating gamma systems for stereotactic radiosurgery. Int J Radiat Oncol Biol Phys. 1999, 43:689-696. 10.1016/S03603016(98)00428-3

5. Kubo HD, Araki F: Dosimetry and mechanical accuracy of the first rotating gamma system installed in North America. Med Phys. 2002, 29:2497-2505. 10.1118/1.1514039

6. Shaw E, Kline R, Gillin M, Souhami L, Hirschfeld A, Dinapoli R, Martin L: Radiation Therapy Oncology Group: radiosurgery quality assurance guidelines. Int J Radiat Oncol Biol Phys. 1993, 27:1231-1239. 10.1016/0360-3016(93)90548-A

7. Bhatnagar JP, Novotny J, Niranjan A, Kondziolka D, Flickinger J, Lunsford D, Huq MS: First year experience with newly developed Leksell Gamma Knife ${ }^{\circledR}$ Perfexion ${ }^{\mathrm{TM}}$. J Med Phys. 2009, 34:141-148. 10.4103/09716203.54848 